[CONTRIbUtion from the Severance Laboratory of Oberlin College.]

\title{
CELLULOSE NITRATE AS AN EMULSIFYING AGENT.
}

\author{
By Harry N. Holmes and DON H Cameron.
}

Received September 8, 1921.

Two immiscible liquids are readily emulsified temporarily by shaking, but a third substance, the so-called "emulsifying agent" must be present to insure stability to the emulsion. Soaps are the best known emulsifying agents, but other substances are used, even gelatin and basic cupric sulfate. As a general rule the emulsifying agent must be soluble in, or rather, peptized by one of the liquids. This explains the common use of colloids for this purpose, yet a finely divided substance that is more readily wetted by the one liquid than by the other may serve.

Cellulose nitrate is colloidally dispersed in such a number of liquids that it seemed certain it must be an excellent emulsifying agent for certain pairs of immiscible liquids. In addition, its use promised to throw some light on the various theories of emulsification. Schoepf ${ }^{1}$ used it to emulsify glycerol in alcohol-ether.

Choice of Liquids. - Water is generally selected as one of the two liquids in an emulsion but glycerol may be used in its place in most cases. Pairs of immiscible liquids that do not use either water or glycerol are far from numerous. In the conventional phrases "water-in-oil" or "oilin-water" emulsions we class water and glycerol as "water," and most other liquids as "oil."

For this research, the liquids chosen are arranged in pairs. Liquids under $A$ are insoluble or but slightly soluble in the liquids under $B$. Their action toward cellulose nitrate is indicated. Cellulose nitrate is abbreviated as "c. n."

\begin{tabular}{|c|c|c|c|}
\hline \multicolumn{2}{|c|}{ A } & & $\mathrm{B}$ \\
\hline Do not peptize c. $n$. & Peptize c. n. & Indifferent & Precipitate c. $n$. \\
\hline Glycerol & Acetone & $\begin{array}{l}\text { Benzene } \\
\text { Toluene }\end{array}$ & $\begin{array}{l}\text { Carbon disulfide } \\
\text { Carbon tetrachloride } \\
\text { Chloroform }\end{array}$ \\
\hline $\begin{array}{c}\text { Glycerol } \\
\text { or }\end{array}$ & $\begin{array}{l}\text { Ethyl acetate } \\
\text { or }\end{array}$ & Toluene & Chloroform \\
\hline Water & Amyl acetate & Toluene & \\
\hline
\end{tabular}

Peptization of Cellulose Nitrate.-Cellulose nitrate is peptized by acetone, amyl acetate, alcohol-ether, acetic acid, etc. From such solutions it is readily precipitated by any liquid which does not peptize it and which is soluble in the given cellulose-nitrate solvent. For example, carbon disulfide, carbon tetrachloride and chloroform precipitate the nitrate from "solution" in amyl acetate. Water will precipitate it from water-soluble solvents such as acetone, methyl alcohol and acetic acid.

1 Kolloid Z., 8, 83 (1911). 
Benzene and toluene, while they do not peptize the dry ester, can be added in large amounts to solutions of that material in other solvents, without causing precipitation. Possibly these two liquids possess some peptizing power.

Emulsion Types.-Using cellulose nitrate as the emulsifying agent, the emulsions formed were of the "water-in-oil" type. For example, emulsions of water in amyl acetate and of glycerol in acetone were formed. The type was determined by placing a drop of the emulsion upon the liquid of each phase and observing with which liquid it was miscible. An emulsion of water in amyl acetate mixed readily with amyl acetate, indicating that water was the dispersed phase.

Theory of Emulsification.-Cellulose nitrate, peptized in a mixture of 1 part of amyl acetate and 3 parts of benzene, will form the continuous phase for an emulsion with water as dispersed phase. If several large drops of water are added to such a cellulose nitrate solution in a beaker, a film can soon be seen to form around each water drop. These films appear to be of a tough and elastic nature and to interfere with the coalescence of the drops. This is visible evidence to support the theory that a coherent film of the colloidal emulsifying agent is formed around each dispersed drop in an emulsion.

The film which is formed in this case is rendered visible when there is sufficient difference in refractive index between the film and the surrounding liquids. In other emulsions the film may be present but invisible because there may be no such difference in refractive indices.

A visible interfacial film indicates an increased concentration of the emulsifying agent at the liquid-liquid interface. This increase was determined quantitatively in the manner described below.

\section{Experimental Part.}

An emulsion of glycerol dispersed in an acetone solution of cellulose nitrate was selected for this study. This system is one in which water is not used for either phase. Both phases readily dissolve in water while the emulsifying agent remains undissolved.

Reagents.-The glycerol used was a commercial U. S. P. IX quality. The index of refraction of this was observed and found to be 1.4668 at $20^{\circ}$, indicating the presence of about $4-5 \%$ of water.

The acetone was a commercial c. P. grade which had been dried for several days over calcium chloride and further purified by distilling over calcium chloride.

A sample of cellulose nitrate ${ }^{2}$ containing $11.04 \%$ of nitrogen was selected for this work. The solid ester was dried in the air over a steam radiator for several days and a $5 \%$ stock solution prepared by peptizing in acetone.

Procedure.-Acetone was found to be somewhat soluble in glycerol, so the glycerol for the dispersed phase was first saturated with acetone.

a Obtained through the courtesy of the E. I. Du Pont de Nemours Co. 
Seven cellulose nitrate-acetone solutions were prepared from the stock solution, each with a different concentration of the nitrate. These solutions were used for the continuous phase in a set of emulsions prepared as follows.

Twenty-five cc. of cellulose nitrate-acetone solution was measured with a pipet into a $125 \mathrm{cc}$. emulsion bottle of $35 \mathrm{~mm}$. diameter. Forty cc. of glycerol was added, with 5 minutes' shaking in a power-driven shaking machine after each addition. Twenty-five minutes' additional shaking was given after the final addition of glycerol, in order to produce a maximum and uniform disintegration of the glycerol into minute drops.

The shaking machine was of such a size that 7 of the emulsion bottles could be shaken at one time. A set of 7 emulsions, each with acetone solution of different cellulose nitrate content, was prepared. The 7 emulsions received the same mechanical treatment and were in all ways subjected to uniform conditions.

After being prepared this set was put aside to "cream." In these emulsions, since the glycerol has a greater density than the acetone, the cream layer sinks, leaving a clear acetone-cellulose nitrate solution at the top, with a sharp boundary between the two layers.

After "creaming" had taken place, $10 \mathrm{cc}$. of the clear acetone layer above was removed and the cellulose nitrate content determined, and compared with that of the original cellulose nitrate-acetone solution.

Determination of Cellulose Nitrate Content.-Several different methods were tried and the following finally chosen as a satisfactory means of accurately determining the cellulose nitrate content of an acetone solution. The difficulty encountered in this determination was that of removing the small amount of glycerol retained by the acetone.

Ten cc. of the acetone solution was withdrawn with a pipet, the same pipet being used in each case. This was put into a $100 \mathrm{cc}$. beaker and about $50 \mathrm{cc}$. of water added, causing some of the nitrate to go into a coarse colloidal suspension while the remainder was precipitated in curdy form. Two drops of a $10 \%$ solution of aluminum chloride were then added to coagulate the suspended matter, and the whole heated to boiling for a few minutes to eliminate the acetone and aid coagulation. The precipitated material was then filtered on a weighed Gooch crucible, washed with water and dried to a constant weight at $90-100^{\circ}$. Care was taken to prevent decomposition of the material from overheating. The cellulose nitrate contents of both the stock solution and the solutions from the emulsions were determined in this manner.

Experimental Results.-After the set of 7 emulsions had stood undisturbed for 18 hours, $10 \mathrm{cc}$. of the clear acetone layer was removed and the cellulose nitrate content determined. The exact content in the original solutions was also accurately measured. The values of Table I were observed.

By comparison of the values obtained in Determinations I and II on the stock solutions it is seen that the method gave results accurate to the third decimal place. 
TABLE I

RESULTS

Stock solution

$\begin{array}{ccc}\begin{array}{c}\text { Average } \\ \text { G. }\end{array} & \begin{array}{c}\text { From emulsion } \\ \text { G. }\end{array} & \begin{array}{c}\text { Loss of Ester } \\ \text { G. }\end{array} \\ 0.0058 & 0.0049 & 0.0009 \\ 0.0274 & 0.0251 & 0.0023 \\ 0.0344 & 0.0310 & 0.0034 \\ 0.0531 & 0.0493 & 0.0037 \\ 0.0988 & 0.0910 & 0.0078 \\ 0.1463 & 0.1300 & 0.0163 \\ \ldots \ldots & 0.2168 & \ldots \ldots\end{array}$

Speed of Creaming and Stability.-The speed of creaming and the stability of the emulsions in the above set were progressive from 1 to 7 . Emulsion 1 creamed very quickly, and in less than an hour started to break. Emulsion 7 creamed very slowly, and after 18 hours the acetone layer was but little more than $10 \mathrm{cc}$. in volume This emulsion-that is, the cream layer in the bottledid not break for more than a week. Between these extremes the creaming and breaking while progressive as to order, showed some variations in regard to time. Emulsions 1,2, 3 and 4 were comparatively unstable, breaking in less than 3 days. Emulsions 5, 6 and 7 were of the same order of stability. They remained unbroken for a week.

It will be noted that these emulsions were made with such proportions of the two liquids that a cream of 70 to $80 \%$ dispersed phase could 10 separate, leaving a clear upper layer of about $15 \mathrm{cc}$. of acetone.

\section{Conclusions.}

The experimental data show that there is an increase in concentration of cellulose nitrate at the acetone-glycerol interface. The values, when plotted, do not give a curve resembling a typical adsorption curve, but rather show some irreversible reaction, as coagulation, in addition to adsorption.

\section{$\uparrow$
50
40
30
20
10}

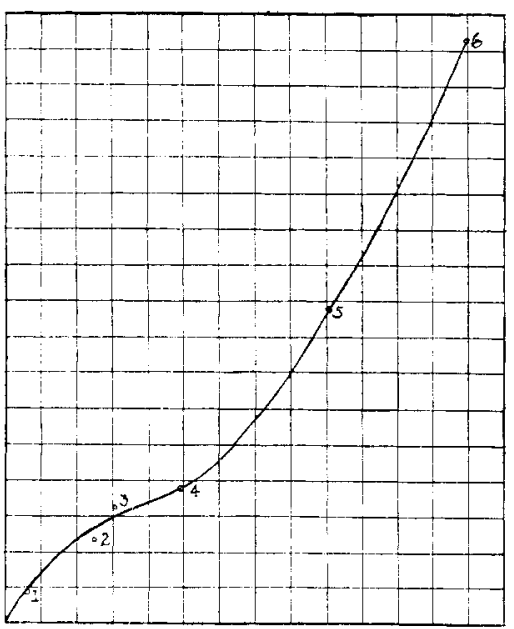

$1020304050 \longrightarrow c$

Adsorption of cellulose nitrate by glycerol at the acetone-glycerol interface.

$\begin{array}{lc}\begin{array}{c}\text { Grams adsorbed } \\ \text { in } 10 \mathrm{cc} .\end{array} & \begin{array}{c}c \\ \text { Grams free } \\ \text { in } 10 \mathrm{cc}\end{array} \\ \text { 1. } 0.0009 & 0.0049 \\ \text { 2. } 0.0023 & 0.0251 \\ \text { 3. } 0.0034 & 0.0310 \\ \text { 4. } 0.0037 & 0.0493 \\ \text { 5. } 0.0078 & 0.0910 \\ \text { 6. } 0.0163 & 0.1300 \\ \text { 7. }- & 0.2168\end{array}$


The nature of the curve is such as would be expected after having seen a visible film of the nitrate form around water drops in an amyl acetatebenzene solution as mentioned above. Between 0 and 4 on the curve there is evidence of adsorption only. Between Points 4 and 5 some radical change occurs and we believe that adsorption is so great that precipitation or coagulation results.

The change in direction of the curve between Points 4 and 5 , since it is the result of a sudden increase in the amount of cellulose nitrate at the acetone-glycerol interface, should correspond to some physical difference between Emulsions 4 and 5. As noted above, emulsions 1, 2, 3 and 4 are much less stable than Emulsions 5, 6 and 7 .

The values and consequently the curve given cannot be claimed as absolutely accurate although the 7 emulsions were made under the same conditions and subjected to identical treatment. It is assumed, however, that the active surfaces in all the emulsions were very nearly the same.

The Interfacial Film.-The stability of any emulsion is due largely to the nature of the interfacial film which is formed. From the above results it seems probable that with cellulose nitrate there is a minimum concentration of the nitrate necessary for the production of a stable emulsion.

The interfacial film may be regarded as an equilibrium product resulting from the precipitating action on the "water" side and the peptizing action on the solvent side. When equilibrium is reached the amount of cellulose nitrate in the film should be in equilibrium with the amount in "solution."

The desirable film for an emulsion is one that forms readily and comes to equilibrium in a short time. It should be elastic, tough, and should change little with age. Such a film on the "solvent" side will be gelatinous and swollen with that liquid, due to peptizing action. On the "water" side, the cellulose nitrate will be coagulated and "wetted" somewhat by the "water" phase.

\section{Summary.}

Cellulose nitrate was found to serve as an excellent emulsifying agent in dispersing water (or glycerol) throughout amyl acetate or other "solvents" for the nitrate.

Visible concentration films of cellulose nitrate were observed around large drops of water "emulsified" in amyl-acetate-benzene. An increase in concentration of cellulose nitrate at the acetone-glycerol interface was studied quantitatively.

The properties of an ideal emulsifying film are discussed.

OberLin, Ohio. 